\title{
Cellular precision of orientation and spatial frequency maps in macaque V1
}

2

Nian-Sheng Ju1*, Shu-Chen Guan²*, Shi-Ming Tang ${ }^{1,2,3 \#, ~ \& ~ C o n g ~ Y u}{ }^{2,3,4 \#}$ 05/04/2019 ${ }^{1}$ School of Life Sciences, ${ }^{2}$ PKU-Tsinghua Center for Life Sciences, ${ }^{3}$ IDG-McGovern Institute for Brain Research, and ${ }^{4}$ Department of Psychology, Peking University, Beijing, China *Equal contribution co-first authors \#Correspondence authors: Shi-Ming Tang (tangshm@pku.edu.cn) \& Cong Yu (yucong@pku.edu.cn)

\section{Abstract}

Functional organization of neuronal response properties along the surface of the neocortex is a fundamental guiding principle of neural computation in the brain. Despite this importance, the cellular precision of functional maps is still largely unknown. We address the challenge by using two-photon calcium imaging to measure cell-specific orientation and spatial frequency (SF) responses across fields of macaque V1 superficial layers. The cellular orientation maps confirm iso-orientation domains, but rarely show pinwheels. Pinwheels obtained through conventional Gaussian smoothing and vector summation of orientation responses mostly overlap with blood vessel regions, suggesting false singularities. Cellular SF maps clarify existing controversies by showing weak iso-frequency clusters, which also suggests a weak geometric relationship between orientation and SF maps. Most neurons are tuned to medium frequencies, but the tuning 
functions are often asymmetric with a wider low- or high-frequency branch, which may help encode low or high SF information for later decoding.

\section{Introduction}

Neurons in primate $\mathrm{V} 1$ are functionally organized according to their preferences to stimulus features (Hubel \& Wiesel, 1962, 1968), likely a result of wiring optimization (Chklovskii \& Koulakov, 2004) or self-organization during evolvement (Kaschube et al., 2010). The local structures of functional maps may be correlated to the tuning properties of neurons (Callaway, 1998; McLaughlin et al., 2003; Nauhaus et al., 2008), such that V1 neurons near the orientation pinwheels tend to have broader tuning than those in iso-orientation domains (Nauhaus et al., 2008). These correlations may suggest the guiding roles of functional organization in neural computation.

Orientation and spatial frequency are two fundamental stimulus features. It is well known that the orientation preferences of V1 neurons are mapped in iso-orientation domains that vary smoothly (Hubel \& Wiesel, 1962, 1963), and that different isoorientation domains converge at singularity points or pinwheels (Bonhoeffer \& Grinvald, 1991). Nevertheless, Hubel and Wiesel (2005) disagreed on the idea of pinwheels from optical imaging experiments because pinwheels are not centered on blobs where neurons have poor orientation tuning, likely an artifact caused by the limited resolution of optical imaging (Polimeni et al., 2005).

V1 spatial frequency maps are less understood and somewhat controversial (Issa et al., 2008; Nauhaus et al., 2012). Single-unit results from monkey V1 and cat area 17 are 
conflicting on whether spatial frequency preferences change smoothly or abruptly (Silverman et al., 1989; Born \& Tootell, 1991; DeAngelis et al., 1999; Molotchnikoff et al., 2007). More recent optical imaging results tend to show clusters of spatial frequency tuning, but are inconsistent on whether these clusters are continuous, pinwheeled, or not repeating at all (Everson et al., 1998; Issa et al., 2000; Xu et al., 2007). To complicate the matter, there is suspicion that the spatial frequency maps from intrinsic optical

mistakenly interpreted as spatial frequency domains (Sirovich \& Uglesich, 2004). Such

vascular artifacts should apply to orientation maps too.

Single-unit recording data may be inaccurate due to under-sampling. Intrinsic 1 optical imaging, on the other hand, lacks cellular resolution. The two-photon imaging 2 technology is able to overcome these difficulties in large measure by simultaneously recording hundreds of neurons' activities at single cell precision (Ohki et al., 2005; Nauhaus et al., 2012; Nauhaus et al., 2016; Li et al., 2017). Most relevant to the current study, Nauhaus et al. $(2012 ; 2016)$ have studied V1 orientation and spatial frequency tuning in anesthetized macaques, using two-photon imaging with a bulk-loaded calcium 7 indicator Oregon Green BAPTA-1 AM (OGB-1). They obtained orientation maps with iso8 orientation domains radiating from pinwheels, as well as spatial frequency maps with 9 continuous iso-spatial frequency domains. However, these maps on the basis of "largescale" imaging are still pixel based and lack single cell details. That is, the orientation and 1 spatial frequency maps show each pixel's, not each cell's, orientation and spatial 2 frequency preferences, respectively. Here the orientation preference of a specific pixel is 
the vector sum of the pixel's responses to 8 orientations, and the spatial frequency preference is the center-of-mass of the pixel's tuning function measured with 6 spatial frequencies.

We decided to use long-term two-photon calcium imaging (Li et al., 2017) to study cellular orientation and spatial frequency maps in awake macaque monkeys. Our setup resolution in an imaging window of $850 \times 850 \mu \mathrm{m}^{2}$, which was large enough to reveal the two-dimensional layouts of orientation and spatial frequency preferences, and was similar in size to the large-scale imaging window of Nauhaus et al. (2012; 2016). Unlike the short imagining time when OGB-1 is used as the calcium indicator, the calcium indicator GCaMP5 makes it possible to image the same neurons' activities for an extended period (Li et al., 2017). For each imaging window we were able to measure at two recording depths $(150$ and $300 \mu \mathrm{m})$ the neuronal responses to a Gabor stimulus at 12 orientations, 6 spatial frequencies, 2 opposite drafting directions, and 3 sizes (to maximize summation and minimize surround suppression), to achieve detailed response mapping. We also used 1 1.5 s inter-stimulus intervals to minimize the interferences of responses from previous stimulus presentations.

\section{Results}

We recorded orientation and spatial frequency responses in V1 superficial layers

1 II/III in four awake macaque monkeys (Figure 1a). Recordings were made at 6 locations 
at depths of 150 and $300 \mu \mathrm{m}$ from the cortical surface, so that 12 sets of data were collected. Initial screening with bandpass filtering and thresholding of differential images $(\Delta \mathrm{F}=\mathrm{F}-\mathrm{F} 0)$ (see Methods) identified 12534 regions of interests (ROIs) or possible cell bodies (Figure 1b). Each cell's responses $(\Delta \mathrm{F} / \mathrm{F} 0)$ were then fitted with a Gaussian model to estimate orientation tuning, and a Difference-of-Gaussian model to estimate spatial frequency tuning. A total of 11225 cells (89.6\%) were tuned to orientation and/or spatial frequency, and were included in data analysis (examples shown in Figure 1d, e). Among them, 6874 (61.2\%) were tuned to both orientation and spatial frequency, $1924(17.1 \%)$ to orientation only, and 2427 (21.6\%) to spatial frequency only.

a

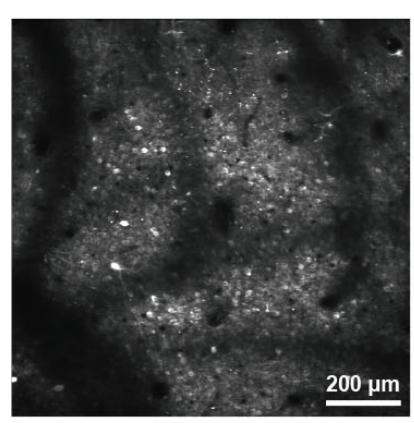

d

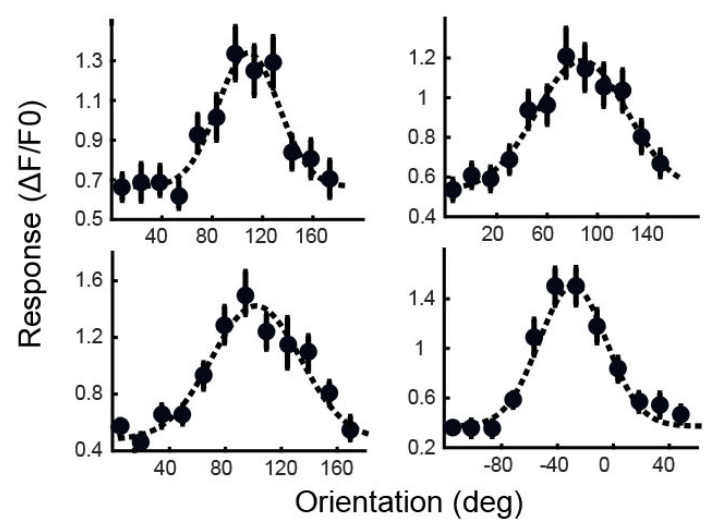

b

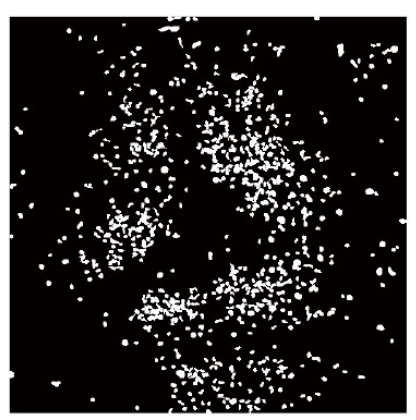

e
C

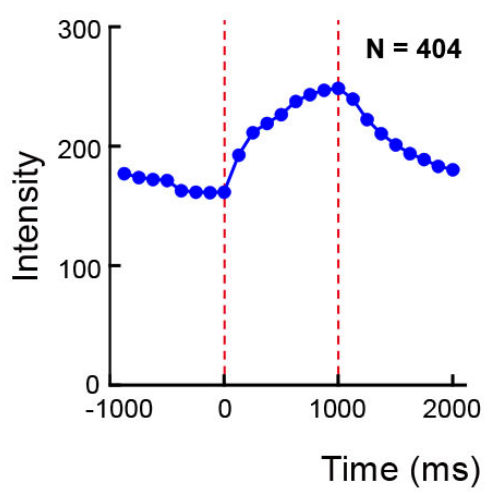

Figure 1. Two-photon calcium imaging and orientation and spatial frequency tuning functions. 
a. The average two-photon image after alignment correction over a recording session at extracted from a after band-pass filtering and thresholding ( $>=3$ standard deviation of the mean). c. The mean time course of calcium responses over all neurons that were tuned to both orientation and spatial frequency at monkey A site 1 at $150 \mu \mathrm{m}$ depth, each at the optimal stimulus orientation and spatial frequency. Two vertical dashed lines indicate stimulus onset and offset. Each dot indicates one frame ( 8 frames per second, $125 \mathrm{~ms}$ per frame). The mean response started to saturate around 5th-6th frames (625-750 ms) after stimulus onset. d. Examples of individual orientation tuning functions fitted with a Gaussian model. e. Examples of individual spatial frequency tuning functions fitted with a Difference-of-Gaussian by blood vessels (Figure 2a). Each patch contained clear clusters of neurons tuned to similar orientations. These iso-orientation domains changed preferred orientations smoothly. Moreover, the distributions of orientations domains appeared similar at 150 and $300 \mu \mathrm{m}$ depths, suggesting vertical orientation columns. These results confirmed previous reports of iso-orientation domains and orientation columns (Hubel \& Wiesel, 1962; Bonhoeffer \& Grinvald, 1991).

To quantify the iso-orientation domains, we calculated the autocorrelations of orientation tuning as a function of absolute cortical distance within the same map. 
Compared to the mean baseline autocorrelations with neurons randomly shuffled (mean $=0.053$ within $800 \mu \mathrm{m}$ ), the measured mean autocorrelations over 12 orientation maps were significantly higher within cortical distances of $0-50 \mu \mathrm{m}, 50-100 \mu \mathrm{m}$, and $100-150$ $\mu \mathrm{m}\left(\mathrm{p}_{\mathrm{s}}<0.001\right.$, two tailed paired t-tests here and later), and became similar within 150$200 \mu \mathrm{m}(\mathrm{p}=0.133)$ (Figure $2 \mathrm{~b})$. The normalized (measured/baseline) autocorrelations were 8.86, 7.14, and 4.04 within 0-50, 50-100, and 100-150 $\mu \mathrm{m}$, respectively. Data from individual maps were quite similar to the mean. These results suggest significant isoorientation domains that were about $150 \mu \mathrm{m}$ in width.

And to quantify the columnar structures of orientation preferences, we calculated the cross-correlations of orientation tuning as a function of absolute cortical distance between maps at two depths, which showed a similar trend. The mean crosscorrelations over all recording sites $(0.43,0.35$, and 0.22 within $0-50,50-100$, and 100 $150 \mu \mathrm{m}$, respectively) were $7.44,6.19,4.22$ times as high as the corresponding baselines (mean $=0.056$ across 3 distances $)\left(\mathrm{p}_{\mathrm{s}}<0.011\right)$, respectively, and became insignificantly different at 150-200 $\mu \mathrm{m}(\mathrm{p}=0.142)$ (Figure 2c). Individual maps showed similar trends, except for the one with monkey A site 2 that showed much poorer alignment between two depths (normalized autocorrelations $=1.67,1.70$, and 1.67 within $0-50,50-100$, and 100-150 $\mu \mathrm{m}$, respectively). Therefore, $\mathrm{V} 1$ neurons in macaques were organized vertically in orientation columns, but with exceptions. 
a
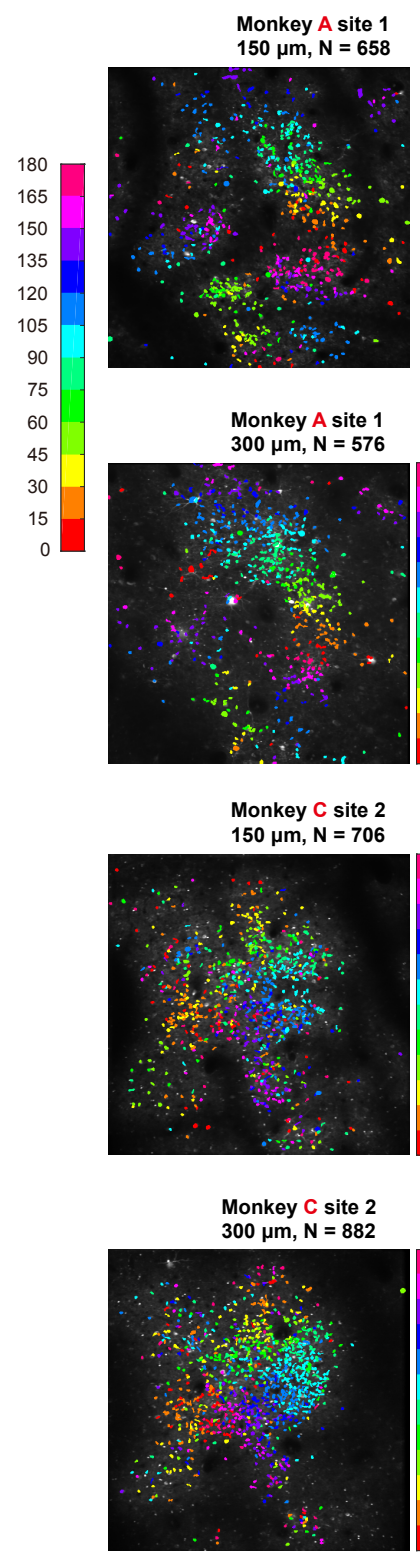
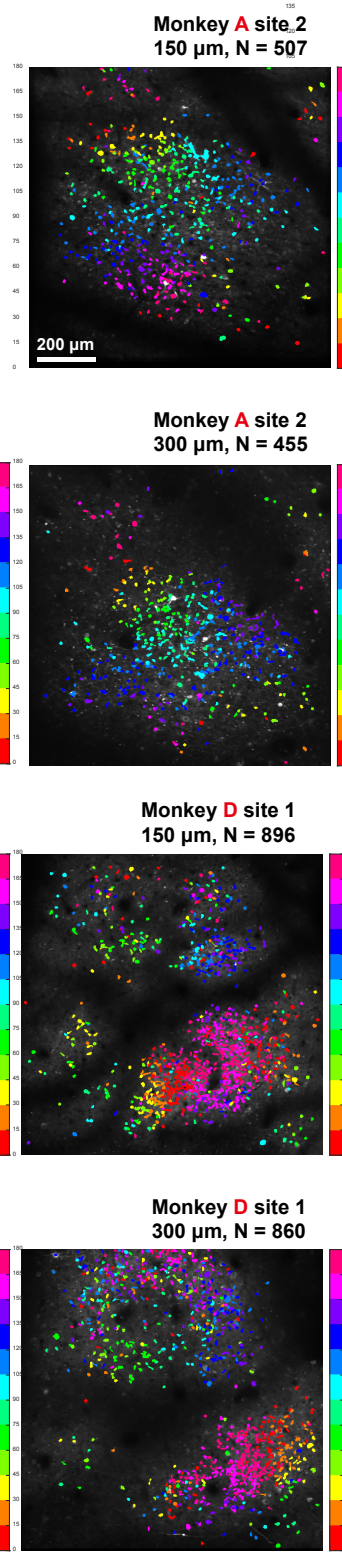
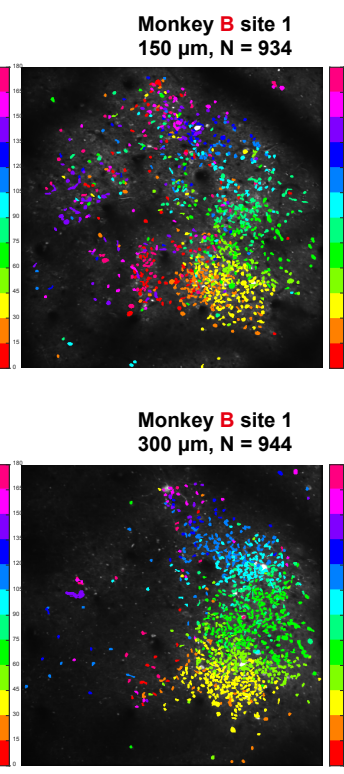

b

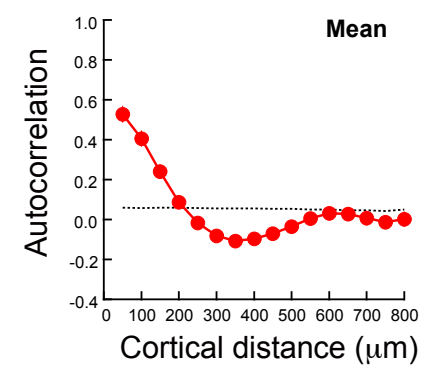

C

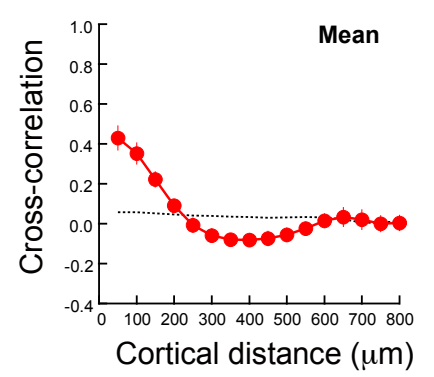

Figure 2. Cellular orientation maps. a. Cellular orientation maps from 4 macaques at two

recording depths. b. Mean within-map autocorrelation of orientation tuning over 12

orientation maps as a function of the absolute cortical distance. Each red dot indicates the

averaged measured autocorrelation within a $50 \mu \mathrm{m}$ bin up to the dot's corresponding cortical distance on the x-axis. The black dashed curve is the baseline autocorrelation simulated with neurons randomly shuffled in positions. c. Mean cross-correlation of orientation tuning between two depths over 6 recording sites as a function of the absolute cortical distance. 
Each red dot indicates the averaged measured cross-correlation within a $50 \mu \mathrm{m}$ bin up to the dot's corresponding cortical distance on the x-axis. The black dashed curve represents the mean baseline cross-correlation. Error bars represent \pm 1 SEM.

What really surprised us in these cellular orientation maps was the rare appearance maps were divided into patches by blood vessels (and likely shallows of more superficial blood vessels). Within each patch, iso-orientation domains rarely appeared to converge in pinwheels (Figure 2a). As pinwheel structures are mostly revealed by optical imaging, we constructed optical imaging-like orientation maps, in which the responses to each orientation were first Gaussian smoothed, and then the orientation preference of each pixel was independently calculated (Figure 3b, example maps of monkey A recording site 1; see Methods). The optical imaging-like orientation maps did show numerous pinwheels (e.g., 4 on the upper map and 5 on the lower map in Figure 3b). However, when cellular orientation maps (Figure 2a) and contours of optical imaging-like maps were overlaid (Figure 3c), it was apparent that all 9 pinwheels were located in regions with few neurons recorded. As more easily discernable in Figure 3a, these pinwheels were either overlaid with radial or vertical blood vessels, or in the shallows of likely more superficial blood vessels.

Pinwheels were also evident in orientation maps through large-scale two-photon imaging (Nauhaus et al., 2012; 2016). These maps were different from our optical imaging-like maps (Figure 3b) in that each pixel's orientation preference was calculated 
without initial Gaussian smoothing of responses to each orientation. We followed Nauhaus et al. $(2012 ; 2016)$ to recreate orientation maps using data sets from monkey A site 1 (Figure 3d). These orientation maps did not show clear boundaries of isoorientation domains as did maps in Nauhaus et al. $(2012 ; 2016)$, but at least some of

regions with possible pinwheels (e.g., solid circles) appeared to correspond to the

pinwheels estimated in Figure 3b, and most of them were actually located in blood-

vessel regions.

Overall, among 50 pinwheels revealed in all 12 optical imaging-like orientation maps, only 2 were likely real pinwheels (indicated by dashing circles in maps of monkey imaging-like orientation maps.

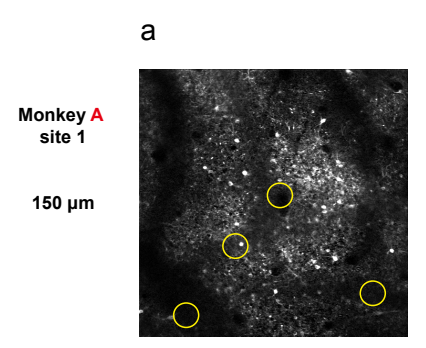

b
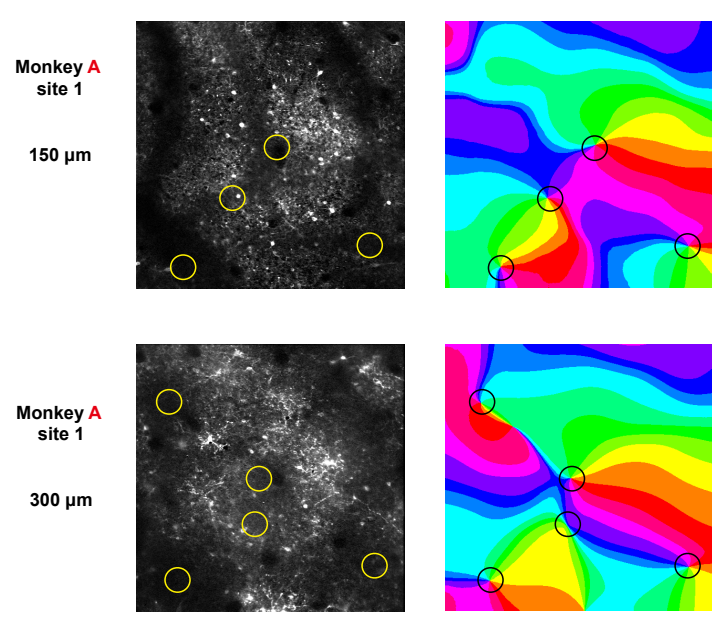

e

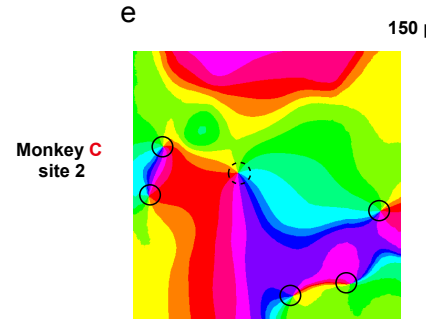

$150 \mu \mathrm{m}$

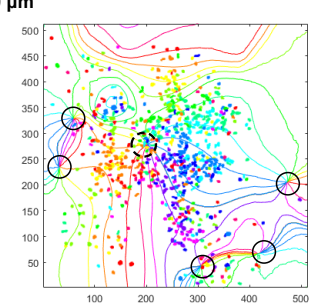

c
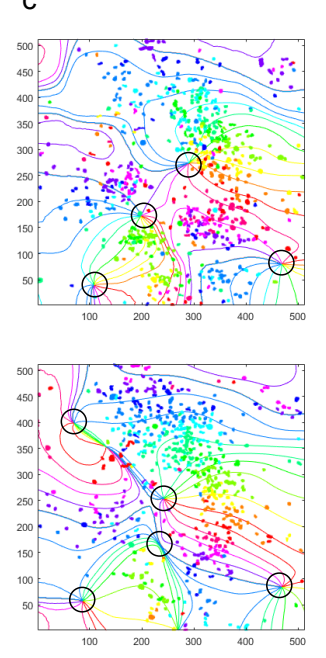

d
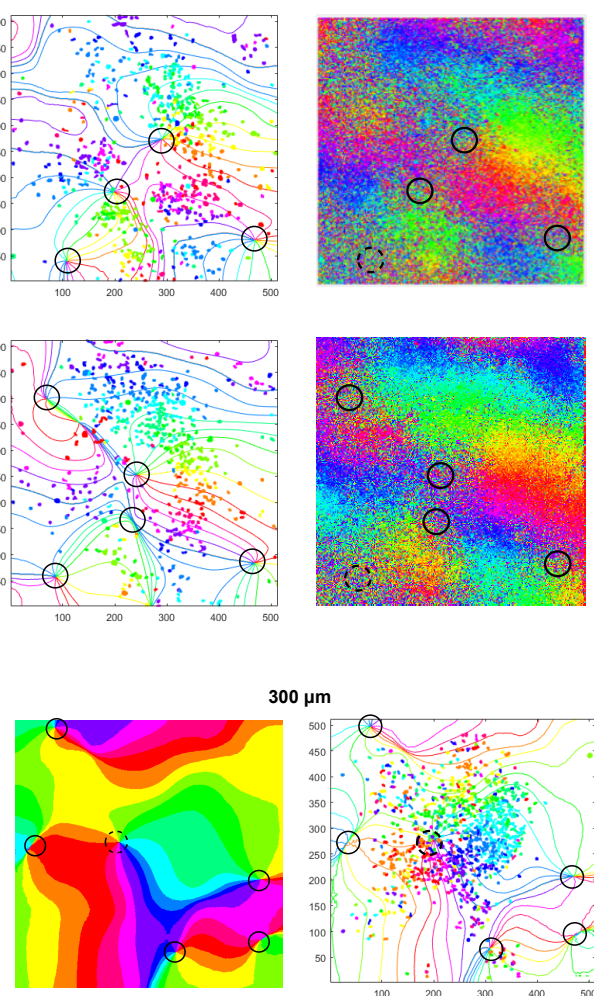

$300 \mu \mathrm{m}$

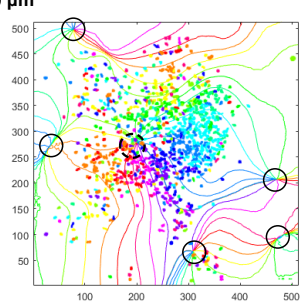


Figure 3. Comparisons of cellular and optical imaging-like orientation maps. a. The twophoton images over a recording session at Monkey A recording site 1 at 150 and $300 \mu \mathrm{m}$ depths. The circles in each map indicate locations of pinwheels revealed from optical imaging-like orientation maps in $\mathbf{b}$. b. Optical imaging-like orientation maps with the data sets of monkey A site 1. c. Overlaid cellular orientation maps of monkey A site 1 from Fig. $2 a$ and contoured optical imaging-like orientation maps from $\mathbf{b}$. $\mathbf{d}$. Orientation maps obtained using the methods of Nauhaus et al. $(2012 ; 2016)$. The same circles in each corresponding map indicate locations of pinwheels in optical imaging-like maps from b . e. Optical imaging-like orientation maps and overlaid cellular and contoured optical imaging-like orientation maps of monkey $\mathrm{C}$ site 2 at $150 \mu \mathrm{m}$ (left two panels) and $300 \mu \mathrm{m}$ (right two panels). The maps at each recording depth contain a possible real pinwheel as indicated by a dashed circle.

\section{Cellular spatial frequency Maps}

The cellular spatial frequency maps showed poor iso-frequency domains (Figure 4a). The mean within-map autocorrelation over 12 maps was $0.67,0.63$, and 0.59 within $0-50,50-100$, and $100-150 \mu \mathrm{m}$, respectively, slightly but significantly higher than the corresponding baselines, all of which at $0.52(\mathrm{p}<0.001)$, and became insignificantly different within $150-200 \mu \mathrm{m}(\mathrm{p}=0.114)$ (Figure $4 \mathrm{~b})$. The normalized autocorrelations were $1.28,1.20$, and 1.12 within $0-50,50-100$, and 100-150 $\mu \mathrm{m}$, respectively, which were close to the chance level at 1 , in sharp contrast to high orientation autocorrelations at 8.86, 7.14, and 4.04 within corresponding cortical distances. 
poor even within the first $100 \mu \mathrm{m}$ of the cortical distance (Figure 4c). The mean measured vs. baseline cross-correlations was 0.55 vs. $0.46(p=0.033)$ and 0.52 vs. 0.45 ( $\mathrm{p}=0.033$ ) within $0-50$ and $50-100 \mu \mathrm{m}$, respectively, with normalized cross-correlations at 1.20 and 1.16, much lower than the orientation cross-correlations of 7.44 and 6.19 within corresponding cortical distances. The difference became insignificant at 100-150 $\mu \mathrm{m}(\mathrm{p}=0.070)$. These data indicated weak columnar structures in spatial frequency maps. attributed to baseline autocorrelations and cross-correlations that were about 10 times or more as high than those with orientation maps ( 0.53 vs. 0.05 with mean autocorrelation and 0.44 vs. 0.03 with mean cross-correlation across all distances up to $800 \mu \mathrm{m})$. The high baselines mostly resulted from a narrow range of preferred spatial frequencies in each map. Take the maps of monkey A site 1 for example (Figure 4d), 81.2\% neurons' preferred spatial frequencies fell into a 2-octave range from 1.01 to 4.04 cpd at $150 \mu \mathrm{m}$, and $92.6 \%$ fell into a 2-octave range from 1.07 to $4.41 \mathrm{cpd}$ at $300 \mu \mathrm{m}$. Moreover, there were far less neurons tuned to spatial frequencies lower than $1 \mathrm{cpd}$ or higher than $4 \mathrm{cpd}$. Other spatial frequency maps also showed similar narrow ranges of preferred spatial frequencies and scarcity of cells tuned to low or high spatial frequencies (Figure 4d). On the average 83.0\% of neurons from all monkeys were tuned to a 2-octave range of spatial frequencies, with $10.6 \%$ tuned to lower frequencies and $6.4 \%$ to higher frequencies. These trends were largely consistent with De Valois et al. (1982) who showed that most V1 neurons' peak frequencies spread over a 2-octave 
range (approximately 1.4-5.6 cpd for Y cells and 1.0-4.0 cpd for X cells) at the comparable eccentricities (3-5 $)$ in anesthetized macaques. The exact ranges of the 2octave range varied among 12 maps. Many were around 1-4 cpd, but a few were either 0.5-1 octaves lower (monkey A site 2, monkey C site 2, and monkey D site 1, all at 150 $\mu \mathrm{m}$; Figure 4d) or higher (monkey B at two depths and monkey D at $300 \mu \mathrm{m}$; Figure 4d). The shifts of spatial frequency ranges were not related to retinal eccentricity. For example, the recording sites of monkeys B and D were not closer to the fovea than the other two monkeys. Rather the shifts might suggest individual differences, or clusters of spatial frequency tuning possibly in a grander scale than orientation clusters. More data are required to verify these possibilities. Nevertheless, none of these maps contained many neurons tuned to spatial frequencies lower than $0.5 \mathrm{cpd}$ or higher than $8 \mathrm{cpd}$. 
a
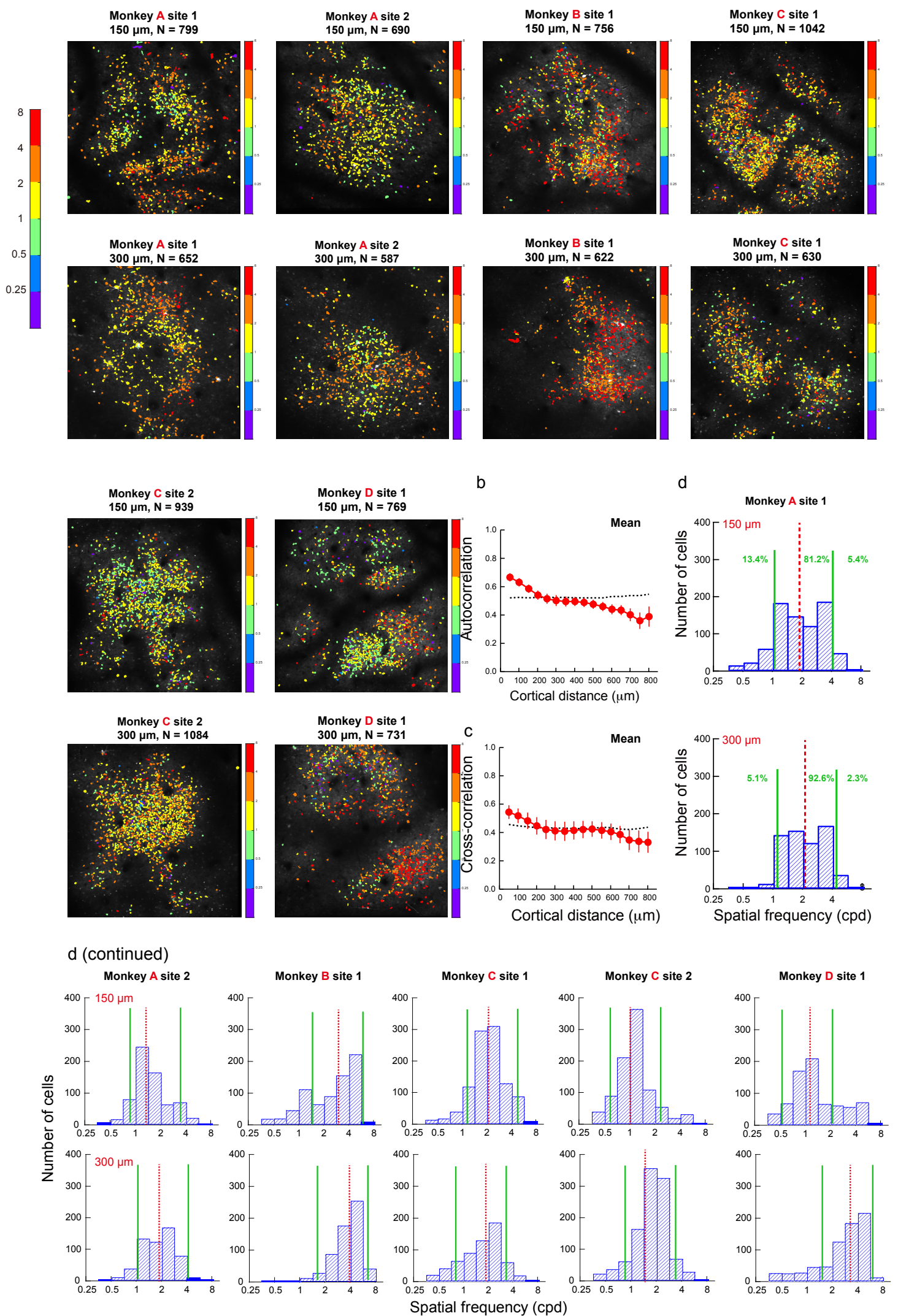

Figure 4. Cellular spatial frequency maps. a. Cellular spatial frequency maps from 4 
frequency maps as a function of the absolute cortical distance. Each red dot indicates the averaged measured autocorrelation within a $50 \mu \mathrm{m}$ bin up to the dot's corresponding cortical distance on the x-axis. The black dashed curve represents the baseline autocorrelation with shuffled neurons. c. Mean cross-correlation of spatial frequency maps between two depths over 6 recording sites as a function of the absolute cortical distance. Each red dot indicates the averaged measured cross-correlation within a $50 \mu \mathrm{m}$ bin up to the dot's corresponding cortical distance on the x-axis. The black dashed curve represents the baseline crosscorrelation with shuffled neurons. d. The distributions of neurons against preferred spatial frequency in half-octave steps in monkey A site 1 at 150 and $300 \mu \mathrm{m}$ depths. The pair of green vertical solid lines indicate the low and high boundaries of a 2-octave range of Behaviorally, macaque monkeys, like humans, are sensitive to low spatial preferred spatial frequencies. The red vertical dashed line indicates the median preferred spatial frequencies. frequencies, as well as to high spatial frequencies up to $\sim 30 \mathrm{cpd}$ at comparable retinal eccentricities (De Valois et al., 1974). How could low and high spatial frequency information be represented in the brain if there are too few V1 neurons tuned to them? On possible strategy is to have these low and high spatial frequencies first encoded by medium-frequency V1 neurons, and then decoded from population responses by downstream neurons. For example, assuming the simplest case in which two neurons tuned to the same medium frequency but have different slopes of low (or high) frequency branches, subtracting two tuning functions would give a differential function 
that contains a lower (or higher) spatial frequency peak.

We calculated the lower- and higher-half bandwidths at half height for the spatial frequency tuning function of each neuron in our dataset, and contrasted the higher-half bandwidth against the lower-half bandwidth for all neurons at 150 and $300 \mu \mathrm{m}$ depths octaves at $300 \mu \mathrm{m}$, about 0.4 octaves wider than the median higher-half bandwidths that were 0.77 octaves at both depths. Therefore, more neurons' spatial frequency tuning functions contained a wider low frequency branch that would encode low frequency information, like those shown in Figure 1e. Still, there were also many neurons whose tuning functions contained a wider high frequency branch to encode high spatial frequency information. Some neurons had a very wider lower-half or higher-half bandwidth of $>2$ octaves. These were mostly low-pass or high-pass neurons that would encode very low or very high (up to cutoff) spatial frequencies.
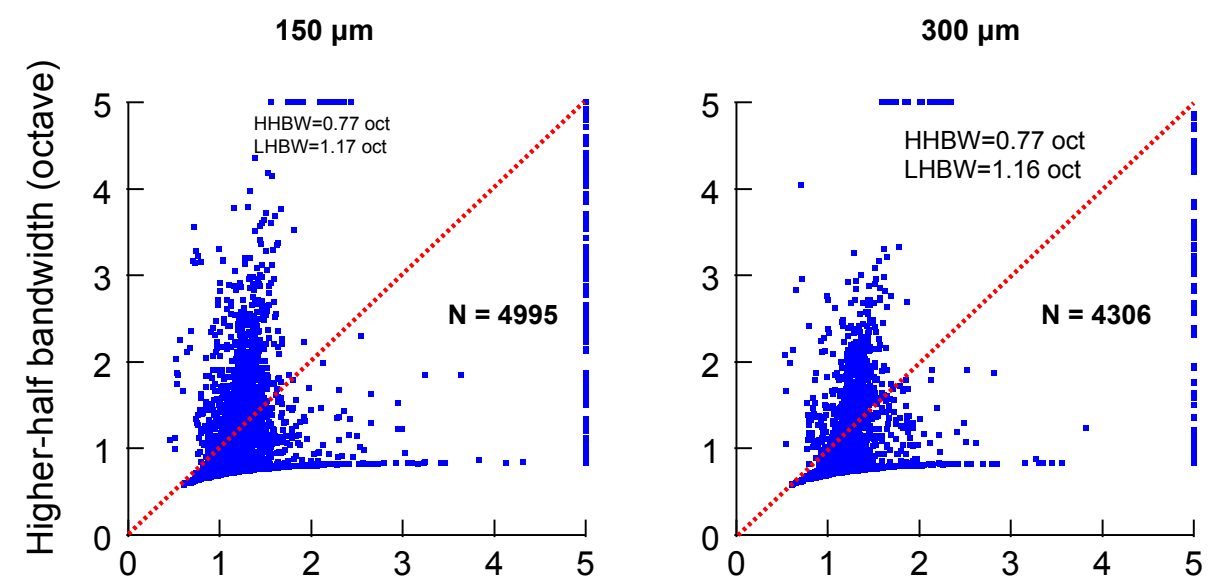

Lower-half bandwidth (octave)

Figure 5. All neuron's lower-half bandwidths (LHBW) and higher-half bandwidths (HHBW) of spatial frequency tuning functions are contrasted at two recording depths. A low-pass neuron's LHBW is assigned to be 5 if not computable. So is a high-pass neuron's HHBW. 


\section{Discussion}

In this study we used two-photon imaging with genetically encoded calcium indicators (GCaMP5) to study cellular orientation and spatial frequency maps in awake macaque V1. Cellular orientation maps confirm iso-orientation domains and orientation columns, but reveal scarce evidence for pinwheels. The orientation maps are divided by

domains rarely converge on singularity points or pinwheels. Instead, many pinwheels

revealed in optical imaging-like orientation maps through Gaussian-smoothing and 1 vector summation of orientation responses tend to be centered on neuron-less blood vessel regions. Although we are not questioning the very existence of orientation pinwheels, indeed there are two likely pinwheels in monkey C (Fig. 3e), as well as in previous two-photon imaging studies (Ohki et al., 2005; Li et al., 2017), our results suggest that real pinwheels could be accidental occurrences in V1 orientation maps, at 6 least at superficial layers where our recordings were performed.

7 The cellular spatial frequency maps reveal weak iso-spatial frequency domains and columnar structures. This finding is consistent with Sirovich and Uglesich (2004) that V1 spatial frequency maps from optical imaging contain no columnar structures after removing the vascular effects. After Gaussian-smoothing and vector summation of the raw spatial frequency responses, we did find in optical imaging-like spatial frequency maps that the blood vessel regions were filled with colors of nearby neurons to form 
false iso-frequency domains. The findings that most neurons are tuned to a narrow range of medium spatial frequencies ( $\sim 2$ octaves), and only a small percentage of neurons are tuned to low and high spatial frequencies, are largely consistent with De Valois et al. (1982), as we mentioned earlier, as well as Edwards et al. (1995) who reported that blob neurons and most interblob neurons in macaque V1 share similar preferred medium spatial frequencies with a low boundary of $1.4 \mathrm{cpd}$, except that some interblob neurons are tuned to higher spatial frequencies. In addition, the narrow range of spatial frequency tuning agrees with a psychophysical masking study that spatial frequency tuning of spatial channels is limited within a 2-octave range (Kontsevich \& Tyler, 2013).

Nevertheless, human and monkeys are sensitive to low and high spatial frequencies. This dilemma could be solved by many middle frequency neurons whose tuning functions either a wider low-frequency branch or a wider high-frequency branch to encode low or high frequency information. Foster et al. (1985) reported in a single-unit study that V2 neurons tend to be tuned to spatial frequencies 2-octave lower than V1 neurons do. At comparable retinal eccentricities $\left(2-5^{\circ}\right)$, the preferred spatial frequencies range from 0.5 to $8.0 \mathrm{cpd}$ in $\mathrm{V} 1$, similar to our data, and are about two octaves lower from 0.2 to $2.1 \mathrm{cpd}$ in V2. It is likely that low spatial frequency information is first encoded by medium frequency V1 neurons, and then decoded by low-frequency V2

It is imperative to compare our two-photon imaging results and those of Nauhaus et 
al. $(2012 ; 2016)$, the only two-photon imaging studies that constructed orientation and spatial frequency maps in macaque V1. Nauhaus et al. (2012; 2016) relied on "largescale" pixel-based imaging to obtain orientation and spatial frequency maps, and "finescale" imaging to measure tuning functions of individual neurons and calculate pairwise correlations. Fine-scale imaging only imaged a $200 \times 200 \mu \mathrm{m}^{2}$ area with a relatively small number of neurons. We were able to obtain cellular orientation and spatial frequency

(1.6 vs. $3.0 \mu \mathrm{m}_{\text {pixel }^{-1}}$ ) and a lot more neurons. For example, the total number of

neurons considered in analysis by Nauhaus et al. (2012) was 735, in contrast to over

11,000 orientation and/or spatial frequency tuned neurons in our study.

Orientation maps $\quad$ The pixel-based orientation maps in Nauhaus et al. (2012;

2016) show iso-orientation domains and pinwheels. Our cellular orientations maps, each divided by blood vessels into various sizes and shapes of patches, also reveal isoorientation domains, but rarely pinwheels. Instead optical imaging-like orientation maps through Gaussian smoothing and vector summation of raw orientation responses led to many false pinwheels located on neuron-less blood vessel regions. The blood-vessel artifacts were not considered by Nauhaus et al. (2012; 2016).

Autocorrelation indicates the degree of functional clustering. Nauhaus et al. (2012) reported that normalized orientation autocorrelation declines gradually from $\sim 8$ to $\sim 1$ within a cortical distance from 7-52 $\mu \mathrm{m}$ to around $200 \mu \mathrm{m}$. Similarly, our data show a decline from $\sim 9$ to $\sim 1$ within comparable ranges of cortical distances. Nauhaus et al.'s autocorrelations were estimated from the entire data set from 10 images, with the 
assumption that neurons in these images together represent an unbiased sample. Our estimates were first obtained from each of 12 images and then averaged.

Spatial frequency maps Hauhaus et al. (2012) found that within cortical distances of 7-52 $\mathrm{mm}$, the normalized autocorrelation is $\sim 3$, weaker than $\sim 8$ with orientation maps. We also found weaker spatial frequency clustering with a similar periodicity, but the cortical distance of $50 \mathrm{~mm}$. Hauhaus et al.'s (2012) maps have a wider range of spatial frequency preferences than ours. The spatial frequency maps from two studies both have a similar low boundary of preferred spatial frequencies at $0.5-1 \mathrm{cpd}$, but Nauhaus et al.'s maps include preferences to higher spatial frequencies at 4-8 cpd. Some of our maps do show tuning to higher spatial frequencies, but the ranges of spatial frequency tuning are not getting wider, but also shifting to higher frequencies (Figure 4a). As a main conclusion, Nauhaus et al. (2012) reported that orientation and spatial frequency maps are orthogonal. However, such a relationship is difficult to demonstrate in our cellular maps because of the very weak iso-spatial frequency domains.

In summary, our cellular orientation maps reveal iso-orientation domains and columnar structures, but hardly pinwheels, in superficial layers of macaque V1. Many pinwheels revealed by optical imaging-like orientation maps actually result from false singularities because of the blood vessel effects, suggesting that functional microorganizations revealed with non-cellular level techniques need to be treated with great caution. Cellular spatial frequency maps show poor functional organization of spatial frequency tuning, largely because most neurons prefer a limited range of medium 
frequencies and are spatially intermixed. However, most of these neurons have asymmetric tuning functions that contain a wider branch toward either low or high spatial frequencies, which may be primarily responsible for encoding low or high spatial frequency information for later decoding.

\section{Methods}

\section{Monkey preparation}

Four macaque monkeys (aged 5-8 years) were used in this study. Each monkey was prepared with two sequential surgeries under general anesthesia and strictly sterile conditions. In the first surgery, a 20-mm diameter craniotomy was performed on the skull over V1. The dura was opened and 100-150 nL AAV1.hSynap.GCaMP5G.WPRE.SV40 (AV-1-PV2478, titer 2.37e13 (GC/ml), Penn Vector Core) was pressure-injected at a depth of $\sim 350 \mu \mathrm{m}$. The dura was then sutured, the skull cap was re-attached with three titanium lugs and six screws, and the scalp was sewn up. After the surgery, the animal was returned to the cage, treated with injectable antibiotics (Ceftriaxone sodium, Youcare Pharmaceutical Group, China) for one week. The second surgery was performed 45 days later. A T-shaped steel frame was installed for head stabilization, and an optical window was inserted onto the cortical surface. More details of the preparation and surgical procedures can be found in Li et al. (2017). The procedures were approved by the Institutional Animal Care and Use Committee at Beijing University.

\section{Behavioral task}


After a ten-day recovery from the second surgery, monkeys were seated in primate chairs with head restraint. They were trained to hold fixation on a small white spot $\left(0.1^{\circ}\right)$ with eye positions monitored by an ISCAN ETL-200 infrared eye-tracking system (ISCAN Inc.) at a 500-Hz sampling rate. During the experiment, a trial with the eye position deviated $2^{\circ}$ from the fixation point was discarded and repeated. For the remaining trials, the eye positions were mostly concentrated around the fixation point. and $0.16^{\circ}, 0.28^{\circ}$, and $97.3 \%$ for monkey D.

\section{Visual stimuli}

Visual stimuli were generated by the ViSaGe system (Cambridge Research Systems) and presented on a 21" CRT monitor with a refresh rate of $80 \mathrm{~Hz}$. Monitor resolution was set at 1280 pixel $\times 960$ pixel. Because of the space and monitor size limits, viewing 3-4 hours.

A drifting square-wave grating (full contrast, 4 cpd spatial frequency, 3 cps temporal distances varied depending on the stimulus spatial frequency $(30 \mathrm{~cm}$ at $0.25 / 0.5 / 1 \mathrm{cpd}$, $60 \mathrm{~cm}$ at $2 \mathrm{cpd}$, and $120 \mathrm{~cm}$ at 4/8 cpd). Imaging of all orientations and spatial each imaging window, which was around $2-4^{\circ}$ eccentricity. For orientation and spatial 
frequency tuning measurements, the stimulus was a high-contrast (0.9) Gabor gating (Gaussian-windowed sinusoidal grating) drifting at 2 cycles/s in opposite directions perpendicular to the Gabor orientation. The Gabor grating varied at 12 equal-spaced orientations from $0^{\circ}$ to $165^{\circ}$ in $15^{\circ}$ steps, and 6 spatial frequencies from 0.25 to $8 \mathrm{cpd}$ in 1 octave steps. It also varied in 3 sizes at approximately $1,1.5$, and 2 octaves (or $\sigma=$ $0.42 \lambda, 0.64 \lambda$, and $0.85 \lambda$ ) at $0.25,0.5$, and $1 \mathrm{cpd}$, and $1.5,2$, and 2.5 octaves (or $\sigma=0.64 \lambda$, $0.85 \lambda$, and $1.06 \lambda)$ at 2,4 , and $8 \mathrm{cpd}$, respectively ( $\lambda=$ wavelength). Our pilot measurements found very strong surround suppression with larger stimuli. Therefore, we decided to use these three stimulus sizes (always $>=1$ octave) at each spatial frequency, so that the best responses of each neuron could be roughly estimated with the most center excitation and least surround suppression.

The stimuli at a specific viewing distance were pseudo-randomly presented. Each stimulus was presented for $1 \mathrm{~s}$, with an inter-stimulus interval of $1 \mathrm{~s}$ (monkeys A, B) or $1.5 \mathrm{~s}$ (monkey C, D) to minimize the interference of responses from previous trials (Fig. 1c). Each stimulus condition was repeated 10 times for monkeys $A, B$ and 12 times for monkeys $\mathrm{C}$, D, with half trials for each opposite direction.

\section{Two-photon imaging}

Two-photon imaging was performed with a Prairie Ultima IV (In Vivo) two-photon microscope (Prairie Technologies) and a Ti:sapphire laser (Mai Tai eHP, Spectra Physics). One or two windows of $850 \times 850 \mu \mathrm{m}^{2}$ were selected in each animal and imaged using 1000-nm femtosecond laser under a $16 \times$ objective lens (0.8 N.A., Nikon) at a resolution 
of $1.6 \mu \mathrm{m} /$ pixel. Fast resonant scanning mode (32 fps) was chosen to obtain continuous images of neuronal activity (8 fps after averaging every 4 frames).

Data were analyzed with customized MATLAB codes. A normalized cross-correlation based translation algorithm was used to reduce motion artifacts (Li et al., 2017). Specifically, a template image was first generated by averaging 1000 successive frames in (1) one imaging session. Two-photon images of one cortical area across days were then corrected and aligned using the template image. After the correction, fluorescence changes were associated with corresponding visual stimuli through the time sequence information recorded by Neural Signal Processor (Cerebus system, Blackrock Microsystem). By subtracting the mean of the 4 frames before stimuli onset $(F O)$ from the average of the 6 th-9th frames after stimuli onset $(F)$ across 5 or 6 repeated trials for the same stimulus condition (same orientation, spatial frequency, size, and drifting direction), the differential images $(\Delta F=F-F 0)$ were obtained. These differential images were then filtered with a band-pass Gaussian filter (size $=10-20$ pixels). Finally, connected subsets of pixels ( $>25$ pixels) with average pixel value $>3$ standard deviations $(\Delta F / F 0)$ was calculated as neuronal responses. Each stimulus condition was presented for 10-12 trials, half for each of two opposite directions. For a specific cell's response to a 
specific stimulus condition, the $\mathrm{FO}_{n}$ of the n-th trial was the average of 4 frames before stimulus onset, and $F_{n}$ was the average of 5th-8th or 6th-9th frames after stimulus onset, whichever was greater. $\mathrm{F} 0_{\mathrm{n}}$ was then averaged across 10 or 12 trials to obtain the baseline $\mathrm{F} 0$ for all trials (for the purpose of reducing variations), and $\Delta \mathrm{F}_{\mathrm{n}} / \mathrm{F} 0=\left(\mathrm{F}_{\mathrm{n}}-\right.$ F0)/F0 was taken as the neuron's response to this stimulus at this trial. A small portion (6.3\%) of the neurons showed direction selectivity. For those neurons, the 5-6 trials at to a particular stimulus. F0 was still averaged over 10-12 trials at two opposite directions. orientation and/or spatial frequency. (1) At each spatial frequency, the optimal stimulus size for producing maximal responses at a specific orientation among all orientations was selected. The response to each orientation was decided at this stimulus size. (2) The optimal spatial frequency and orientation producing the maximal response among all conditions were selected. Then responses to 12 orientations were decided at the optimal spatial frequency, and responses to 6 spatial frequencies were decided at the optimal orientation.

(3) To select orientation tuned neurons, a Kruskal-Wallis test was first performed to test whether a neuron's responses at 12 orientations were significantly different from each other $(\mathrm{p}<0.05)$. For those showing significant difference, the orientation tuning function of each neuron was fitting with a Gaussian model: 
where $R(\theta)$ was the response at orientation theta, free parameters $a_{1}, \theta_{0}, \sigma$, and $b$ were the amplitude, peak orientation, standard deviation of the Gaussian function, and minimal response of the neuron, respectively. Only neurons with goodness of fit $\mathrm{R}^{2}>0.5$ were finally selected as orientation tuned neurons.

(4) Similarly, to select spatial frequency tuned neurons, a Kruskal-Wallis test decided whether a neuron responded differently to different spatial frequencies ( $p<$ 0.05), and data fitting with a Different-of-Gaussian model further filtered-in neurons with $\mathrm{R}^{2}>0.5$ as spatial frequency tuned neurons.

$$
\mathrm{R}(s f)=a_{1} e^{-\left(\frac{s f}{\sigma_{1}}\right)^{2}}-a_{2} e^{-\left(\frac{s f}{\sigma_{2}}\right)^{2}}+b
$$

where R(sf) was a neuron's response at spatial frequency sf, free parameters $a_{1}, \sigma_{1}, a_{2}$,

\section{Optical imaging-like orientation maps} and $\sigma_{2}$ were amplitudes and standard deviations of two Gaussians, respectively, and $b$ was the minimal response among 6 spatial frequencies. low-pass Gaussian filter. Preferred orientation of each pixel on orientation map was then deduced from the vector sum of corresponding response intensities with 12 orientations as following,

$$
R s p=\sum_{k=1}^{12} r s p_{k} \exp \left(2 i \theta_{k}\right)
$$

where $\theta_{k}$ was k-th orientation in radians, and $r s p_{k}$ was the response strength at k-th orientation. Each pixel of the orientation map was then assigned to one of 12 bins according to its orientation preference, denoted by different colors (Figure 3b). 
Autocorrelation: Within each orientation or spatial frequency map, every pair of neurons' correlation coefficient of responses to 12 orientations or 6 spatial frequencies from model fitting were calculated. The correlations of all pairs of neurons separated by a specific range of cortical distance (from $0-800 \mu \mathrm{m}$ in $50 \mu \mathrm{m}$ steps) were then averaged as the autocorrelation of this range of cortical distance. Cross-correlation: Same as autocorrelation except neurons were paired from different maps at 150 and $300 \mu \mathrm{m}$ depths.

\section{Acknowledgments} suggestions during the preparation of this manuscript.

\section{References} arranged in pinwheel-like patterns. Nature, 353(6343), 429-431 
Callaway, E. M. (1998). Local circuits in primary visual cortex of the macaque monkey. Annu Rev Neurosci, 21, 47-74 them? Annu Rev Neurosci, 27, 369-392

De Valois, R. L., et al. (1982). Spatial frequency selectivity of cells in macaque visual cortex. Vision Res, 22(5), 545-559 luminance contrast sensitivity tests of macaque and human observers. Vision Res, $14(1), 75-81$

De Valois, R. L., et al. (1974). Psychophysical studies of monkey vision. 3. Spatial

DeAngelis, G. C., et al. (1999). Functional micro-organization of primary visual cortex: receptive field analysis of nearby neurons. J Neurosci, 19(10), 4046-4064

Edwards, D. P., et al. (1995). Contrast sensitivity and spatial frequency response of primate cortical neurons in and around the cytochrome oxidase blobs. Vision Res, 35(11), 1501-1523 visual cortical areas V1 and V2 of the macaque monkey. J Physiol, 365, 331-363 architecture in the cat's visual cortex. J Physiol, 160, 106-154 visual cortex. Proc Natl Acad Sci U S A, 95(14), 8334-8338

Foster, K. H., et al. (1985). Spatial and temporal frequency selectivity of neurones in

Hubel, D. H., \& Wiesel, T. N. (1962). Receptive fields, binocular interaction and functional

Hubel, D. H., \& Wiesel, T. N. (1963). Shape and arrangement of columns in cat's striate cortex. J Physiol, 165, 559-568 
Hubel, D. H., \& Wiesel, T. N. (1968). Receptive fields and functional architecture of monkey striate cortex. J Physiol, 195(1), 215-243

Hubel, D. H., \& Wiesel, T. N. (2005). Brain and Visual Perception: New York: Oxford Press.

Issa, N. P., et al. (2008). Models and measurements of functional maps in V1.J

Issa, N. P., et al. (2000). Spatial frequency maps in cat visual cortex. J Neurosci, 20(22),

Kaschube, M., et al. (2010). Universality in the evolution of orientation columns in the visual cortex. Science, 330(6007), 1113-1116 revealed by sustained perifoveal stimuli. J Vis, 13(1), 22

Kontsevich, L. L., \& Tyler, C. W. (2013). A simpler structure for local spatial channels

Li, M., et al. (2017). Long-Term Two-Photon Imaging in Awake Macaque Monkey. Neuron,

$$
\text { 93(5), 1049-1057 e1043 }
$$

McLaughlin, D., et al. (2003). Large-scale modeling of the primary visual cortex: influence of cortical architecture upon neuronal response. J Physiol Paris, 97(2-3), $237-252$

Molotchnikoff, S., et al. (2007). Spatial frequency characteristics of nearby neurons in cats' visual cortex. Neurosci Lett, 418(3), 242-247

Nauhaus, I., et al. (2008). Neuronal selectivity and local map structure in visual cortex. Neuron, 57(5), 673-679 
Nauhaus, I., et al. (2012). Orthogonal micro-organization of orientation and spatial frequency in primate primary visual cortex. Nat Neurosci, 15(12), 1683-1690

Ohki, K., et al. (2005). Functional imaging with cellular resolution reveals precise microarchitecture in visual cortex. Nature, 433(7026), 597-603

Polimeni, J. R., et al. (2005). Physical limits to spatial resolution of optical recording: clarifying the spatial structure of cortical hypercolumns. Proc Natl Acad Sci U S A, 102(11), 4158-4163

Silverman, M. S., et al. (1989). Spatial-frequency organization in primate striate cortex. Proc Natl Acad Sci U S A, 86(2), 711-715

Sirovich, L., \& Uglesich, R. (2004). The organization of orientation and spatial frequency in primary visual cortex. Proc Natl Acad Sci U S A, 101(48), 16941-16946 